\title{
A box model of circulation and melting in ice shelf caverns
}

\author{
Dirk Olbers and Hartmut Hellmer \\ Alfred Wegener Institute for Polar and Marine Research, 27515 Bremerhaven, Germany \\ Ocean Dynamics, revised November 23, 2009
}

\begin{abstract}
A simple box model of the circulation into and inside the ocean cavern beneath an ice shelf is used to estimate the melt rates of Antarctic glaciers and ice shelves. The model uses simplified cavern geometries and includes a coarse parameterization of the overturning circulation and vertical mixing. The melting/freezing physics at the ice shelf/ocean interface are those usually implemented in high resolution circulation models of ice shelf caverns. The model is driven by the thermohaline inflow conditions and coupling to the heat and freshwater exchanges at the sea surface in front of the cavern. We tune the model for Pine Island Glacier and then apply it to six other major caverns. The dependence of the melting rate on thermohaline conditions at the ice shelf front is investigated for this set of caverns, including sensitivity studies, alternative parameterizations, and warming scenarios. An analytical relation between the melting rate and the inflow temperature is derived for a particular model version, showing a quadratic dependence of basal melting on a small temperature difference between the inflow and the grounding zone which changes to a linear dependence for larger differences.

The model predicts melting at all ice shelf bases in agreement with observations, ranging from below a meter per year for Ronne Ice Shelf to about $25 \mathrm{~m} / \mathrm{y}$ for the Pine Island Glacier. In a warming scenario with a one degree increase of the inflow temperature the latter glacier responds with a 1.4 fold increase of the melting rate. Other caverns respond by more than a ten fold increase, as e.g. Ronne Ice Shelf. The model is suitable for use as simple fast module in coarse large-scale ocean models.
\end{abstract}

\section{Introduction}

Precipitation deposited on the Antarctic ice sheet is released as freshwater to the Southern Ocean either locally due to basal melting of floating ice shelves or remotely due to melting of drifting icebergs (Jacobs et al. 1992). Extensive caverns underlying the ice shelves which represent $\sim 11 \%$ of the ice sheet area and about $50 \%$ of the Antarctic coastline. Local freshwater input significantly impacts the stability of the shelf water column and hence sea ice thickness with far-reaching consequences for water mass characteristics and deep water export (Hellmer 2004). Changes in magnitude of local freshwater input can be assumed 
for the near future in view of the current rates of ice shelf decay at the Antarctic Peninsula (e.g. Skvarca et al. 1999, Braun et al. 2008).

Faster than previously anticipated, ice streams feeding the ice shelves respond to changes in the ice shelf geometry (De Angelis and Skvarca 2003), thus increasing the mass loss of the Antarctic ice sheet and contributing to sea level rise. Satellite observations reveal that various ice streams of the West Antarctic Ice Sheet (WAIS) are thinning (Wingham et al. 2006) and accelerating (Rignot 2008). Since this happens in concert with a retreat of the grounding lines, researchers suggest that the enhanced mass loss of WAIS is caused by a warmer ocean. Indeed, in the Amundsen and Bellingshausen Seas Circumpolar Deep Water reaches the ice shelf fronts with temperatures above $+1^{\circ} \mathrm{C}$ (Hellmer et al. 1998) and can proceed, possibly without substantial cooling, into the deep interior. A 'warm tub' environment is supposed to exist underneath Pine Island Glacier (PIG) where different techniques infer melting near the grounding line in excess of $40 \mathrm{~m} / \mathrm{y}$ (Rignot and Jacobs, 2002, Payne et al. 2007). The relatively simple geometry of the PIG cavern invites the application of numerical models of different complexity (Hellmer et al. 1998, Payne et al. 2007, Thoma et al. 2008) which confirm the high melt rates in the deep interior and provide a mean basal melting of up to $20 \mathrm{~m} / \mathrm{y}$. However, it is still a matter of debate, with consequences for climate warming and related SLR, whether the dependency of the basal mass loss on the external ocean temperature is simply linear or of higher order (Holland et al. 2008). In addition, due to uncertainties in sea floor topography and ice shelf draft, modeled values still bear huge errors. Therefore it remains speculative whether the ocean could drive the collapse of an ice sheet.

The latest IPCC (2007) report emphasizes the lack of understanding of the processes controlling ocean-ice interaction and thus the inability to accurately predict future sea level rise. This statement fostered international efforts to develop coupled ocean-ice shelf-ice sheet models (D. Vaughan, pers. communication 2008), and to consider basal processes in climate models. However, especially the fast-melting ice shelves in the Amundsen and Bellingshausen Seas (Rignot and Jacobs 2002) are too small to be resolved by the present coupled climate models. In the present study, we develop a simple, 'low-cost' box model for the thermohaline circulation beneath an ice shelf, including a state-of-the-art presentation of the processes at the ocean-ice shelf interface, and study its performance for a number of prominent caverns with very different environments and geometries. For observed shelf water characteristics feeding the sub-ice shelf circulation our model provides realistic melt rates, outflow characteristics, and a first guess on the circulation strength from which flushing rates can be inferred. Therefore, climate models representing well the conditions on the Antarctic continental shelf could adapt this box model to circumnavigate an IPCC (2007) claimed deficiency. The simple model, as a stand-alone code, is useful to estimate melt rates from a few observations in front of the ice shelf.

The caverns investigated in the present study and treated by our model are listed in Table 1. The entries for 'basal melting' are taken from the cited literature, and are given the area mean melt rates. The model physics and governing equations are developed in section 2. Section 3 presents the model results for the seven caverns of Table 1. The 'melt law', i.e. the dependence of the melt rate on the external temperature, is derived in section 
4. Finally, a conclusion discusses our model, its implications, and further use.

\begin{tabular}{l|l|cc|cl} 
& & length $L$ & width $W$ & $\begin{array}{c}\text { basal } \\
\text { melting }\end{array}$ & study \\
& & & & & \\
\hline Amery & AMY & 540 & 130 & 0.35 & Williams et al. (2001), M \\
Filchner & FIL & 620 & 160 & 0.35 & Grosfeld et al. (1998), M \\
Fimbulisen & FIM & 200 & 150 & 1.93 & Smedsrud et al. (2006), M \\
Getz & GTZ & 70 & 600 & unknown & \\
Pine Island Glacier & PIG & 70 & 40 & $24-29$ & Payne et al. (2007), M \\
Ronne & RON & 620 & 540 & 0.15 & Joughin and Padman (2003), O \\
Ross & ROS & 800 & 600 & 0.13 & Dinniman et al. (2007), M
\end{tabular}

Table 1: Length and width [in $\mathrm{km}]$, and melt rates [in $\mathrm{m} / \mathrm{y}$ ] of some cavern systems. The geometric parameters are taken from the literature as given by the last column (O: observation, M: model).

\section{Cavern circulation physics in a box model}

Melting of ice in an ice shelf cavern is the consequence of advection of relatively warm water from the shelf ice front to the ice shelf base located at a deeper level where, due to a higher pressure, the parcel's temperature exceeds the local freezing point temperature and its heat content may partly be used for melting. Hence, in addition to advection and mixing physics, a model of the circulation in an ice shelf cavern has to implement melting/freezing physics at the ocean-ice shelf interface. Our aim is to establish these physical processes in a box model with very low resolution and a minimum of the physical ingredients. Because the parcel on its path upward along the ice shelf base may encounter a pressure level where its temperature drops below the local freezing point and freezing of its freshwater content starts, at least three boxes in the cavern are needed: a deep one for the deep inflow, one box for the melting regime at the deep ice shelf base, and a box for the possible freezing regime closer to the ice shelf front (they are denoted by ' $d$ ', ' $w$ ' and ' $i$ ', respectively, see Figure 1). Our box model has two additional boxes ' 1 ' and '2' in the open ocean immediately adjacent to the ice shelf front. The system is driven by the thermohaline fluxes of heat and freshwater into the top box '1', and by a reservoir of heat and salt in the open ocean, denoted by ' 0 '. Except for this extremely coarse resolution, the box model's physics are those implemented in high resolution circulation models of cavern systems (e.g. Payne et al. 2007, Dinniman et al. 2007).

Forcing The system is governed by the balances of the heat and salt content of the respective boxes. The coupling to the offshore heat/salt reservoir 0 is assumed entirely advective in an upstream sense: if, for instance, the flow is southward at depth, i.e. into 

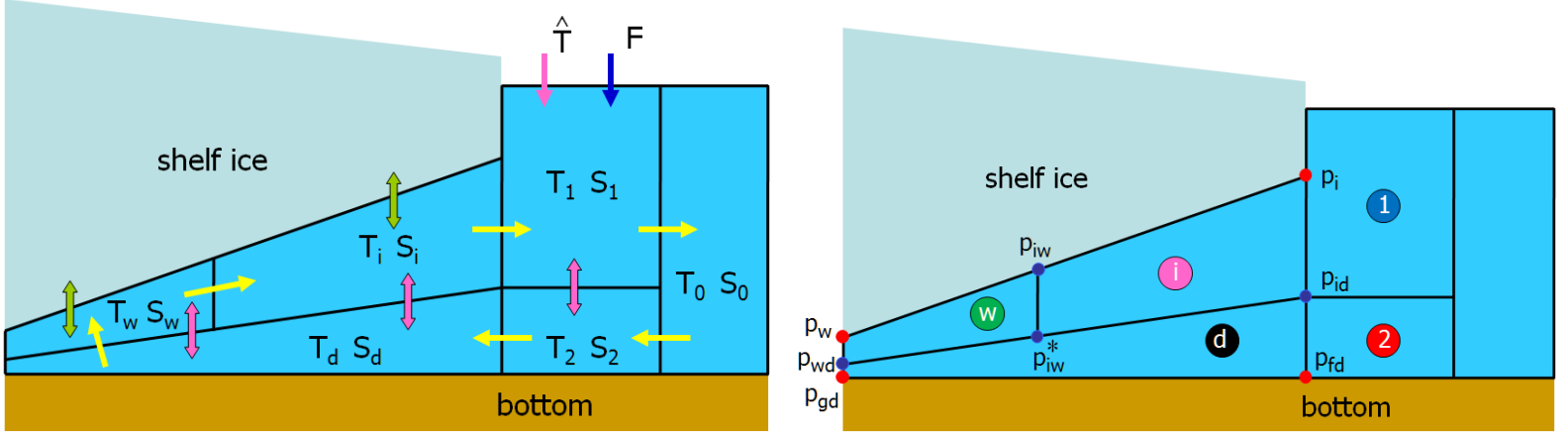

Figure 1: Left: The model geometry of a cavern with advective and diffusive fluxes indicated. Boxes are: $0=$ northern reservoir (passive), $1=$ upper ocean front box, $2=$ deep ocean front box, $d=$ deep cavern box, $w=$ grounding line box, $i=i$ ce shelf water box. Each has a volume $V_{j}$, horizontal area $A_{j}$ and width $W$ which is the same for all boxes. Yellow arrows: volume flux $q$ (positive for clockwise circulation), purple arrows: relaxation $(\gamma)$ and diffusive $(\kappa)$ fluxes, green arrows: melting/freezing, blue arrow: salt flux F. Right: the figure indicates the names of the box coordinates. Red points are the coordinates given in Table 3 for various caverns around Antarctica, with $p \equiv$ depth. Blue points are coordinates determined as described in section 3 and also shown in Table 3.

the cavern, there is a heat flux ${ }^{1} q T_{0}$ and a salt flux $q S_{0}$ into box 2 . Here $q>0$ is the volume transport (unit $\mathrm{m}^{3} \mathrm{~s}^{-1}$ ) into the box which by mass conservation is the same in the entire advection loop (see Figure 1). The outflow of the cavern is into the offshore box 1 which is open to the ocean surface and couples the system to the growth and decay of sea ice. However, coupling for the case $q>0$ only occurs via interfacial mixing with the deep front box 2 . If the stratification in box 1 and box 2 is stable, the mixing is small and the influence of the particular surface forcing in box 1 is only marginal. The simulations presented in this study operate mostly in this regime. We assume a heat flux of the restoring form $\gamma\left(\hat{T}-T_{1}\right)$ into the box 1 with a restoring time scale $\gamma A_{1} / V_{1}=\gamma / H_{1}$ and a temperature $\hat{T}$ which is the freezing temperature $-1.88^{\circ} \mathrm{C}$ at the surface. Here, $A_{k}$ denotes the surface area, $H_{k}$ the depth, and $V_{k}$ the volume of box $k=1,2, i, w, d$. The freezing/melting and export of sea ice induces a (negative/positive) freshwater flux into box 1 which we convert to a (positive/negative) salt flux $F$.

Advection and mixing For $q>0$ the heat and salt contents of box 1 are governed by

$$
\begin{aligned}
& V_{1} \dot{T}_{1}=q\left(T_{i}-T_{1}\right)-A_{1} \kappa\left(T_{1}-T_{2}\right)-A_{1} \gamma\left(T_{1}-\hat{T}\right) \\
& V_{1} \dot{S}_{1}=q\left(S_{i}-S_{1}\right)-A_{1} \kappa\left(S_{1}-S_{2}\right)+A_{1} F
\end{aligned}
$$

\footnotetext{
${ }^{1}$ The common factor $\rho c_{p}$ is canceled in all 'heat' balances.
} 
where we have inserted a term describing vertical mixing between the boxes 1 and 2 . The diffusivity is $\kappa=K_{v} / h$ with the appropriate depth scale $h$. A corresponding term with opposing sign is included in the budget of box 2. The diffusive exchange of the upper box with the deep ocean box should include the condition of static stability. Thus, $\kappa$ is large (i.e. it has a small time scale) when the density difference $\rho_{1}-\rho_{2}$ between the upper box and the deep ocean is above zero (or a small density $\delta \rho \ll \rho$ ), and it is likely that convection will occur, reflecting a statically unstable situation ${ }^{2}$. It is small otherwise. Hence

$$
\kappa=\left\{\begin{array}{llr}
\kappa_{\ell} & \text { if } \rho_{1}-\rho_{2} \leq \delta \rho & \text { stable } \\
\kappa_{h} & \text { otherwise } & \text { unstable }
\end{array}\right.
$$

with $\delta \rho \geq 0$ and $\kappa_{\ell} \ll \kappa_{h}$. For simplicity we assume a linear equation of state, $\rho / \rho_{*}=$ $1-\alpha\left(T-T_{*}\right)+\beta\left(S-S_{*}\right)$ and take $T_{*}=0{ }^{\circ} \mathrm{C}, S_{*}=34$ psu and $\rho_{*}=1033 \mathrm{kgm}^{-3}$. The expansion coefficients, given in Table 2 below, are appropriate for a depth of about $1000 \mathrm{~m}$.

The fluxes and coefficients $q, A F, A \kappa$ and $A \gamma$ all have the unit $\mathrm{m}^{3} \mathrm{~s}^{-1}$. However, whereas $F, \kappa$ and $\gamma$ are determined by fixed coefficients (which may vary between different model integrations), the circulation rate $q$ is a dynamical quantity. As customary in box models with thermohaline ingredients, we assume that the flow is driven by the difference of densities, and here we take the difference between the ice shelf front and the grounding line. We generally take a linear dependence

$$
q=C\left(\rho_{2}-\rho_{w}\right)=C \rho_{*}\left(\beta\left(S_{2}-S_{w}\right)-\alpha\left(T_{2}-T_{w}\right)\right)
$$

with a constant ${ }^{3} C$ and the linear approximation of the equation of state (note that the densities in the above parameterization must be adjusted to the same pressure level). In section 4 a nonlinear extension of (4) will be considered. We should mention that in general $S_{2}>S_{w}, T_{2}>T_{w}$ and $\rho_{2}>\rho_{w}$ in the steady state, hence $q>0$ as depicted in Figure 1. The haline part of the density dominates at low temperatures, and thus the circulation is generally haline-driven.

In fact, the relation (4) is Stommel's pipe law (Stommel 1961): the transport of the early box models of the thermohaline circulation is assumed to occur in connecting pipes, and the density difference between the boxes causes a pressure drop at depth which accelerates the fluid in the pipes against friction. Hence, $C$ depends on the friction coefficients of the system. For non-rotating dynamics it is easy to derive the balance of the meridional overturning circulation, and one finds that diffusion of vorticity, e.g. $A_{v} v_{z z z}$ where $v$ is the meridional velocity, $z$ the vertical coordinate and $A_{v}$ the viscosity, is balanced by the meridional torque $g \rho_{y}$ exerted by the density field ( $y$ is the along-cavern coordinate and $g$ the gravitational acceleration). Then we obtain $q \sim \rho_{y} / A_{v}$ times geometric coefficients (for details see the appendix in Olbers and Zhang 2008). The situation in a rotating system is more complicated but parameterizations of pressure gradients as suggested by Wright et

\footnotetext{
${ }^{2}$ In the simulations discussed in this study the unstable regime only occurred in transient states. The ultimate steady states are determined by the low $\kappa$.

${ }^{3}$ The parameter $C$ has the dimension $\mathrm{m}^{3} \mathrm{~s}^{-1}$ per density unit, i.e. $\mathrm{m}^{6} \mathrm{~kg}^{-1} \mathrm{~s}^{-1}$. In the following we give values for $C$ leaving out the units and the factor $10^{6}$.
} 


\begin{tabular}{c|rc|r} 
parameter & \multicolumn{3}{|r}{ parameter } \\
\hline & & & \\
$\rho_{e}$ & $920 \mathrm{kgm}^{-3}$ & $\rho_{*}$ & $1033 \mathrm{kgm}^{-3}$ \\
$\gamma_{T}$ & $5 \times 10^{-5} \mathrm{~ms}^{-1}$ & $\gamma_{S}$ & $2 \times 10^{-6} \mathrm{~ms}^{-1}$ \\
$c_{p}$ & $3974 \mathrm{Jkg}^{-1} \mathrm{~K}^{-1}$ & $L$ & $3.34 \times 10^{5} \mathrm{Jkg}^{-1}$ \\
$a$ & $-0.057{ }^{\circ} \mathrm{C} / \mathrm{psu}$ & $b$ & $0.0832^{\circ} \mathrm{C}$ \\
$c$ & $7.64 \times 10^{-4}{ }^{\circ} \mathrm{Cdbar}^{-1}$ & $\hat{T}$ & $-1.88^{\circ} \mathrm{C}$ \\
$\alpha$ & $7.5 \times 10^{-5} \mathrm{~K}^{-1}$ & $\beta$ & $7.7 \times 10^{-4}$
\end{tabular}

Table 2: Typical values of some parameters of the cavern system. $\gamma_{T}$ and $\gamma_{S}$ values are taken from Jenkins et al. (2001). Except for the values of the $\gamma$ 's we regard this set of parameter values as fixed.

al. (1998) lead to similar expressions (also here we refer to the discussion in Olbers and Zhang 2008). In the cavern system we bravely model these effects of friction, rotation, and also bottom formstress by the one coefficient $C$.

Melting physics The heat and salt balances at the ocean/ice shelf interface are formulated in close accordance with Hellmer and Olbers (1989). We consider the heat and salt balances of a layer situated immediately below the shelf ice base. Storage of the heat and salt content in the respective layer is ignored for simplicity (the layer has a tiny extent), and thus, fluxes at the lower and the upper interfaces are in balance. Diffusion of heat and salt through the ice is neglected. The heat balance is thus given by $Q_{T}=Q_{T b}$ where $Q_{T}$ is the total heat flux crossing the lower interface and $Q_{T b}$ is the one across the upper interface due to melting/freezing. We parameterize $Q_{T}$ by the difference of temperature between the ice shelf base $\left(T_{b}\right)$ and the ocean $(T)$ in the form $Q_{T}=\rho_{*} c_{p} \gamma_{T}\left(T_{b}-T\right)$ where $\rho_{*}$ and $c_{p}$ are the density and the heat capacity of sea water, respectively, and $\gamma_{T}$ is a turbulent exchange coefficient for heat (in $\mathrm{ms}^{-1}$ ). The amount of heat, $Q_{T b}$, lost by the ocean due to melting of ice $(m>0)$ or gained due to the formation of ice crystals within the layer $(m<0)$, is given by $Q_{T b}=-\rho_{e} L m$ where $\rho_{e}$ is a mean density for ice, $L$ is the latent heat of fusion, and $m$ is the melt rate. Likewise, the total salt flux at the lower interface $Q_{S}=\rho_{*} \gamma_{S}\left(S_{b}-S\right)$ must be equal to the salt flux $Q_{S b}=-\rho_{e} S_{b} m$ caused by meltwater input (or a salt input due to salt rejection during freezing). Here $\gamma_{S}$ is a turbulent salt exchange coefficient and $\left(S_{b}-S\right)$ is the salinity difference between the ice shelf base and the ocean. The parameters $\rho_{\star}, \rho_{e}, c_{p}$ and $L$ are assumed constant (see Table 2 ).

Finally, we assume that the temperature $T_{b}$ at the ocean/ice shelf boundary is at the in-situ freezing point. In a linearized version it can be expressed as a function of the salinity $S_{b}$ and the pressure $p$ by

$$
T_{b}=a S_{b}+b-c p
$$



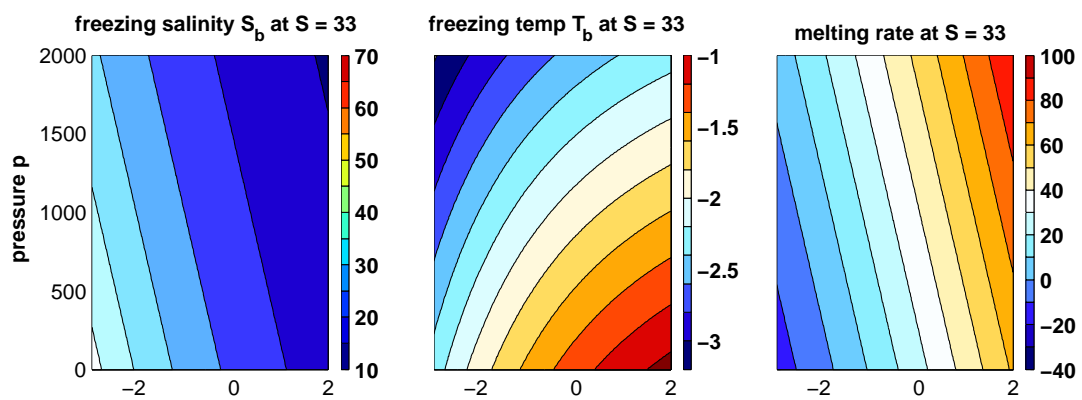

Figure 2: Dependence of the salinity $S_{b}$, freezing temperature $T_{b}$, and melting rate $m$ on pressure $p$ and the ambient temperature $T$ for $S=33$ psu. The melting rate has the unit $\mathrm{m} / \mathrm{y}$.

with the constants $a, b$ and $c$ (see Table 2). To summarize, we have three equations, i.e. (5) and

$$
\begin{aligned}
\gamma_{T}\left(T_{b}-T\right) & =-\nu \lambda m \\
\gamma_{S}\left(S_{b}-S\right) & =-\nu S_{b} m
\end{aligned}
$$

We use the abbreviations $\lambda=L / c_{p} \sim 84 \mathrm{~K}$ and $\nu=\rho_{e} / \rho_{*} \sim 0.89$. The above threeequations model can be solved for the ice shelf base salinity $S_{b}$, temperature $T_{b}$, and the melting rate $m$ in terms of the water properties $T$ and $S$ and the pressure $p$ at the shelf ice base. We find a quadratic equation for the salinity $S_{b}=S_{b}(S, T, p)$, determined by

$$
a S_{b}^{2}-(T-b+c p+d) S_{b}+d S=0
$$

with $d=\lambda \gamma_{S} / \gamma_{T}$. The temperature $T_{b}(S, T, p)$ then follows from (5) and the melting rate $m(S, T, p)$ may be computed from either of (6). The dependence of these quantities is displayed in Figure 2 (upper row). Note that $S_{b}$ is very different from the ocean salinity $S$ outside the turbulent boundary layer. Likewise, the freezing point $a S+b-c p$, computed for the bulk salinity $S$ differs substantially from $T_{b}$. This model of the turbulent boundary layer is applied to the boxes $w$ and $i$; the above quantities $T, S, T_{b}, S_{b}$ and $m$ then get the corresponding index $w$ or $i$.

Finally, we obtain the fluxes $Q_{T}$ and $Q_{S}$ as functions of $T_{w}, S_{w}$ and $p=p_{w}$, the values for box $w$. The water modification in this box is thus governed by

$$
\begin{aligned}
& V_{w} \dot{T}_{w}=q\left(T_{d}-T_{w}\right)-A_{w} \kappa\left(T_{w}-T_{d}\right)+A_{w}\left(\gamma_{T}+m_{w}\right)\left(T_{b w}-T_{w}\right) \\
& V_{w} \dot{S}_{w}=q\left(S_{d}-S_{w}\right)-A_{w} \kappa\left(S_{w}-S_{d}\right)+A_{w}\left(\gamma_{S}+m_{w}\right)\left(S_{b w}-S_{w}\right)
\end{aligned}
$$

This form of the balances takes care of the in- or decrease of the box volume as consequence of the melting or freezing at the ice shelf base (the $m_{w}$ contributions, see Jenkins et al. 2001). For melt rates exceeding $10 \mathrm{~m} / \mathrm{y}$ this implies a substantial correction in the salt balance. 
The governing equations We assume three more active boxes (named $2, d$ and $i$ ) in the same spirit as for the front box 1 and the base box $w$ (see Figure 1). The balances for heat and salt (for $q>0$ ) for the cavern system described above are

$$
\begin{aligned}
\dot{T}_{1} & =q_{1}\left(T_{i}-T_{1}\right)-\kappa_{1}\left(T_{1}-T_{2}\right)-\left(T_{1}-\hat{T}\right) / \tau \\
\dot{S}_{1} & =q_{1}\left(S_{i}-S_{1}\right)-\kappa_{1}\left(S_{1}-S_{2}\right)+F_{1} \\
\dot{T}_{2} & =q_{2}\left(T_{0}-T_{2}\right)+\kappa_{2}\left(T_{1}-T_{2}\right) \\
\dot{S}_{2} & =q_{2}\left(S_{0}-S_{2}\right)+\kappa_{2}\left(S_{1}-S_{2}\right) \\
\dot{T}_{w} & =q_{w}\left(T_{d}-T_{w}\right)-\kappa_{w}\left(T_{w}-T_{d}\right)+\left(\gamma_{T w}+\tilde{m}_{w}\right)\left(T_{b w}-T_{w}\right) \\
\dot{S}_{w} & =q_{w}\left(S_{d}-S_{w}\right)-\kappa_{w}\left(S_{w}-S_{d}\right)+\left(\gamma_{S w}+\tilde{m}_{w}\right)\left(S_{b w}-S_{w}\right) \\
\dot{T}_{i} & =q_{i}\left(T_{w}-T_{i}\right)-\kappa_{i}\left(T_{i}-T_{d}\right)+\left(\gamma_{T i}+\tilde{m}_{i}\right)\left(T_{b i}-T_{i}\right) \\
\dot{S}_{i} & =q_{i}\left(S_{w}-S_{i}\right)-\kappa_{i}\left(S_{i}-S_{d}\right)+\left(\gamma_{S i}+\tilde{m}_{i}\right)\left(S_{b i}-S_{i}\right) \\
\dot{T}_{d} & =q_{d}\left(T_{2}-T_{d}\right)+\left(V_{i} / V_{d}\right) \kappa_{i}\left(T_{i}-T_{d}\right)+\left(V_{w} / V_{d}\right) \kappa_{w}\left(T_{w}-T_{d}\right) \\
\dot{S}_{d} & =q_{d}\left(S_{2}-S_{d}\right)+\left(V_{i} / V_{d}\right) \kappa_{i}\left(S_{i}-S_{d}\right)+\left(V_{w} / V_{d}\right) \kappa_{w}\left(S_{w}-S_{d}\right)
\end{aligned}
$$

where $q_{k}=q / V_{k}, \kappa_{k}=\kappa / H_{k}$ and $\tau=H_{1} / \gamma, F_{1}=F / H_{1}, \gamma_{T w}=\gamma_{T} / H_{w}, \tilde{m}_{w}=m_{w} / H_{w}$ etc. If the circulation reverses $(q<0)$, the advection terms change accordingly, e.g. box 1 is fed by the offshore reservoir instead of box 2. All coefficients now have the dimension $\mathrm{s}^{-1}$. We consider the geometric dimensions for each cavern as fixed (see Table 3 below), likewise most of the coefficients listed in Table 2 parameterizing the melting process, and the temperature $\hat{T}$ of the frontal surface layer, so that the changeable parameters are $C, \kappa_{\ell}, \kappa_{h}, \gamma$ and the salt forcing $F_{1}$ of box 1 . We express the ratio $\kappa_{h} / \kappa_{\ell}=\mathcal{O}\left(10^{4}\right)$ as a fixed large number and model $\kappa_{\ell}=K_{v} / h$ with a diffusivity $K_{v}$ and $h=100 \mathrm{~m}$. As mentioned before, the value of $\kappa_{h}$ is mostly irrelevant. Finally, the salt flux into box 1 enters as $F_{1}=f_{0} S_{*} / H_{1}$ where $f_{0}$ is the local sea ice freezing rate in $\mathrm{ms}^{-1}$. The free parameters are then $C, K_{v}, F_{1}$ and $\tau$. Solutions are obtained by time stepping using a code from MATLAB.

\section{Melting in some cavern systems}

We finalize our study by applying the cavern box model to a suite of ice shelf caverns, namely Amery (AMY), Filchner (FIL), Fimbulisen (FIM), Getz (GTZ), Pine Island Glacier (PIG), Ronne (RON), and Ross (ROS). The basic geometry of these caverns is given by a few coarse estimates of length and width scales in Table 1 and the depths $p_{f d}, p_{g l}, p_{i}$ and $p_{w}$ at the cavern fronts and the grounding lines in Table 3 (see Figure 1 for the locations of the geometric points in the cavern). Rough values for the watermass temperatures and salinities at the front of these caverns are found in Table 4 . We will use these values to set up the box model and adjust the free parameters $C, K_{v}, F_{1}$ and $\tau$ for each cavern to obtain estimates for the melting and overturning rates.

We take a very simple proportionate algorithm to determine the volume $V_{k}$ and the horizontal area $A_{k}(k=1,2, i, w, d)$. The front boxes 1 and 2 have the depths $H_{1}$ and $H_{2}$ 


\begin{tabular}{c|rrrrrrrrrrrr} 
cavern & $p_{i}$ & $p_{i w}$ & $p_{w}$ & $p_{i d}$ & $p_{i w}^{*}$ & $p_{w d}$ & $p_{f d}$ & $p_{g d}$ & $H_{2}$ & $H_{i}$ & $H_{w}$ & $H_{d}$ \\
\hline & & & & & & & & & & & & \\
AMY & 200 & 1400 & 2000 & 400 & 1577 & 2166 & 800 & 2500 & 400 & 188 & 172 & 366 \\
FIL & 400 & 933 & 1200 & 633 & 1122 & 1366 & 1100 & 1700 & 466 & 211 & 177 & 400 \\
FIM & 200 & 266 & 300 & 300 & 433 & 500 & 500 & 900 & 200 & 133 & 183 & 300 \\
GTZ & 300 & 766 & 1000 & 466 & 866 & 1066 & 800 & 1200 & 333 & 133 & 83 & 233 \\
PIG & 400 & 733 & 900 & 600 & 822 & 933 & 1000 & 1000 & 400 & 144 & 61 & 233 \\
RON & 200 & 733 & 1000 & 266 & 800 & 1066 & 400 & 1200 & 133 & 66 & 66 & 133 \\
ROS & 240 & 280 & 300 & 293 & 320 & 333 & 400 & 400 & 106 & 46 & 36 & 86
\end{tabular}

Table 3: Geometric values of the cavern systems, computed from the values in Table 1 and the algorithm explained in the text. Units are dbar or $\mathrm{m}$. Note that $H_{1} \equiv p_{i d}$.

\begin{tabular}{|c|c|c|c|c|c|c|c|c|c|c|c|c|}
\hline cavern & $\min T_{1}$ & $\max T_{1}$ & $\min S_{1}$ & $\max S_{1}$ & $\min T_{2}$ & $\max T_{2}$ & $\min S_{2}$ & $\max S_{2}$ & $\min T_{i}$ & $\max T_{i}$ & $\min S_{i}$ & $\max S_{i}$ \\
\hline AMY & -2.0 & -1.5 & 34.2 & 34.5 & -1.9 & -1.7 & 34.5 & 34.6 & -2.5 & -2 & 34.3 & 34.5 \\
\hline FIL & -1.9 & -1.8 & 34.35 & 34.6 & -2 & -1.95 & 34.68 & 34.74 & -2.25 & -1.9 & 34.6 & 34.65 \\
\hline FIM & -1.8 & -1.7 & 34.2 & 34.35 & -1.84 & 1.8 & 34.34 & 34.36 & -2.1 & -1.9 & 34.23 & 34.32 \\
\hline GTZ & -1.74 & -1.37 & 34.04 & 34.1 & 0.53 & 0.71 & 34.55 & 34.64 & & & & \\
\hline PIG & -1.8 & -0.6 & 33.8 & 34.3 & 1 & 1.2 & 34.64 & 34.7 & -0.4 & 0 & 34.2 & 34.5 \\
\hline RON & -1.9 & -1.5 & 34.3 & 34.6 & -1.9 & -1.5 & 34.65 & 34.85 & -2.15 & -1.9 & 34.5 & 34.8 \\
\hline ROS & -1.9 & -1.85 & 34.66 & 34.78 & -1.9 & -1.5 & 34.75 & 34.9 & -2.11 & -1.95 & 34.64 & 34.72 \\
\hline
\end{tabular}

Table 4: Temperatures and salinities appropriate to minimum and maximum in the boxes 1, 2, and $i$, according to observations from sections along the ice shelf front and other data, taken from the literature. AMY: Wong et al. (1998), FIL: Grosfeld et al. (2001), FIM: Nicholls et al. (2006), GTZ: M. Schröder, pers. communication, PIG: Hellmer et al. (1998), RON: Nichols et al. (2003), ROS: Jacobs and Giulivi (1998).

and horizontal lengths $L_{1}$ and $W$, with $L_{1}=300 \mathrm{~km}$ being identical for all cavern systems but different $W$. Referring to Figure 1 we equate $H_{1}+H_{2} \equiv p_{f d}$ with the water depth at the cavern front and assume $H_{1} \equiv p_{i d}=p_{i}+\xi_{f}\left(p_{f d}-p_{i}\right)$ with proportion factor $\xi_{f}$. Similarly, $p_{w d}=p_{w}+\xi_{g}\left(p_{g d}-p_{w}\right)$ for the depth of the upper box at the grounding line, and furthermore $L_{w}=\eta L_{d}, L_{i}=L_{d}-L_{w}$ for the horizontal lengths of the boxes $w$ and $i$. The side areas $B_{k}$ of the respective rectangles for boxes 1 and 2 and the trapezoids for the boxes $w, i$ and $d$ are then readily calculated, the volumes are $V_{k}=B_{k} W$ and the horizontal areas $A_{k}=L_{k} W$ (neglecting the slope of the interior boxes). Finally, we define an equivalent depth $H_{k}=B_{k} / L_{k}=V_{k} / A_{k}$ for all boxes. The proportion factors are taken $\xi_{f}=\xi_{g}=\eta=1 / 3$, and the resulting geometric quantities are listed in Table 3, using the width, length and depth values from Table 1 and Table 3.

The PIG cavern We start our study of the ensemble of seven caverns with some simulations for the PIG configuration. The thermohaline conditions at the front of PIG are displayed in Figure 3 (from Hellmer et al. 1998). The deep inflow with temperatures above $1^{\circ} \mathrm{C}$ and salinities above $34.64 \mathrm{psu}$ are clearly visible in the deep trench below $800 \mathrm{~m}$ depth 

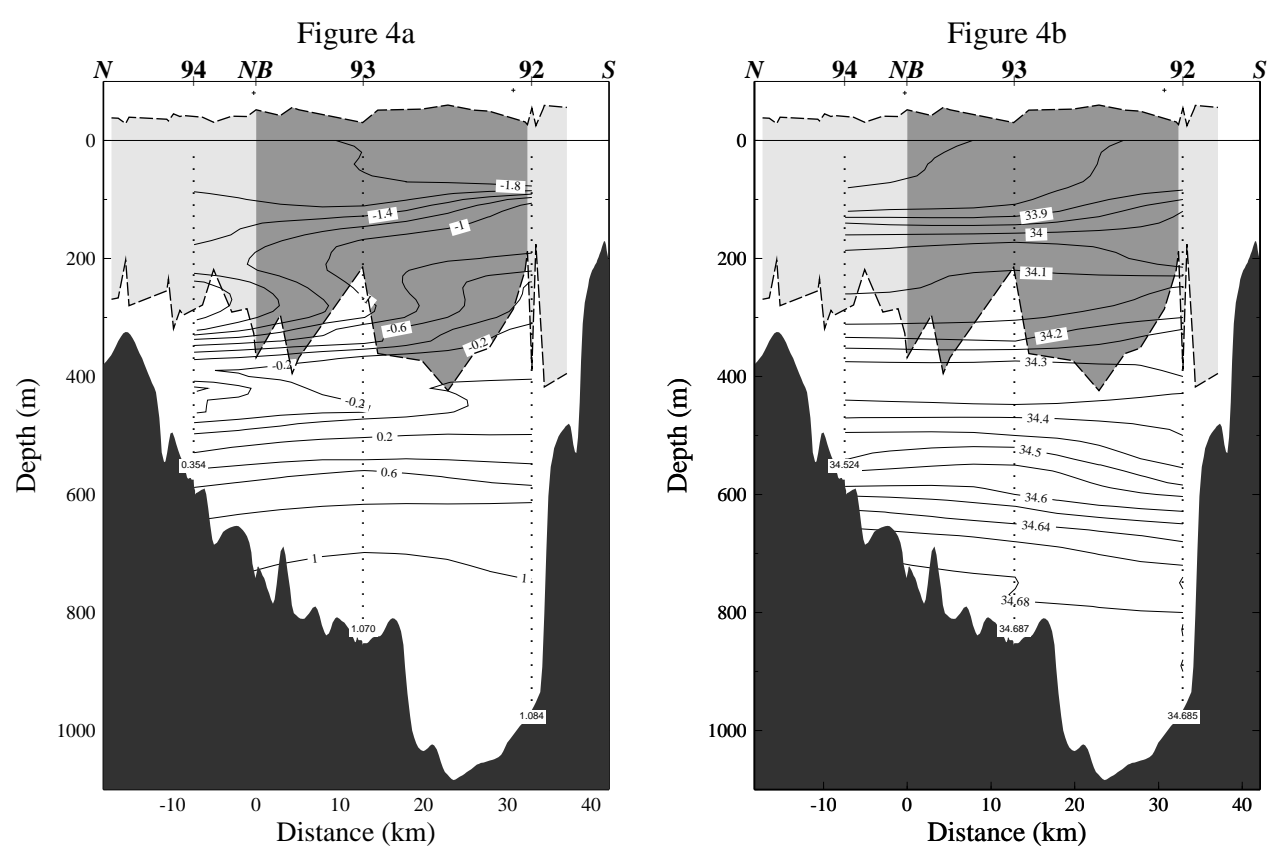

Figure 3: Potential temperature and salinity along the calving front of Pine Island Glacier (light gray) in mid-March 1994 (from Hellmer et al. 1998). The contour interval for temperature is $0.2^{\circ} \mathrm{C}$ and for salinity it changes from 0.05 for $S<34.6$ psu to 0.02 for $S>34.6$ psu.

(to be compared to our box 2 values). The outflow of glacial meltwater (to be compared to our box $i$ values) is seen in the temperature section between about $400 \mathrm{~m}$ and $600 \mathrm{~m}$ depth with $T$ below $-0.2{ }^{\circ} \mathrm{C}$, with a minimum of $-0.4{ }^{\circ} \mathrm{C}$ and $S$ in the range 34.3 to 34.4 psu. Finally, our box 1 should be compared with the hydrographic state at the front above the ice draft, i.e. in the gray shaded domain of the panels of Figure 3. Here, the temperature ranges between -1.8 to $-0.6^{\circ} \mathrm{C}$ and the salinity between 33.8 and $34.2 \mathrm{psu}$. These values are summarized in Table 4 (and correspondingly for the six other caverns). As a further measure of the PIG characteristics we should yield an area mean melting rate of about 20 m/y (e.g. Payne et al. 2007).

We regard this glacier as ideal for parameter studies because it has a simple configuration (its geometry is not too far from our box system), it is likely without any freezing areas at the ice shelf base and has a quasi-permanent fast ice area in front. From the latter condition we suspect that the effect of sea ice freezing is small, hence $F_{1}=0$ can be assumed as a good first guess. Furthermore, the coupling of the interior to the freezing point layer at the front box 1 may be of minor importance, hence $\tau$ can be assumed to be large.

In Figure 4 (upper panels) the case $C=2, K_{v}=10^{-5} \mathrm{~m}^{2} \mathrm{~s}^{-2}, \tau=300$ days and $F_{1}=0$ is shown to yield a moderately acceptable fit to the observed hydrography with overturning and melt rates of $q=0.24 \mathrm{~Sv}$ and $m_{m}=38.6 \mathrm{~m} / \mathrm{y}$. A change of $\tau$ can repair deficits in 

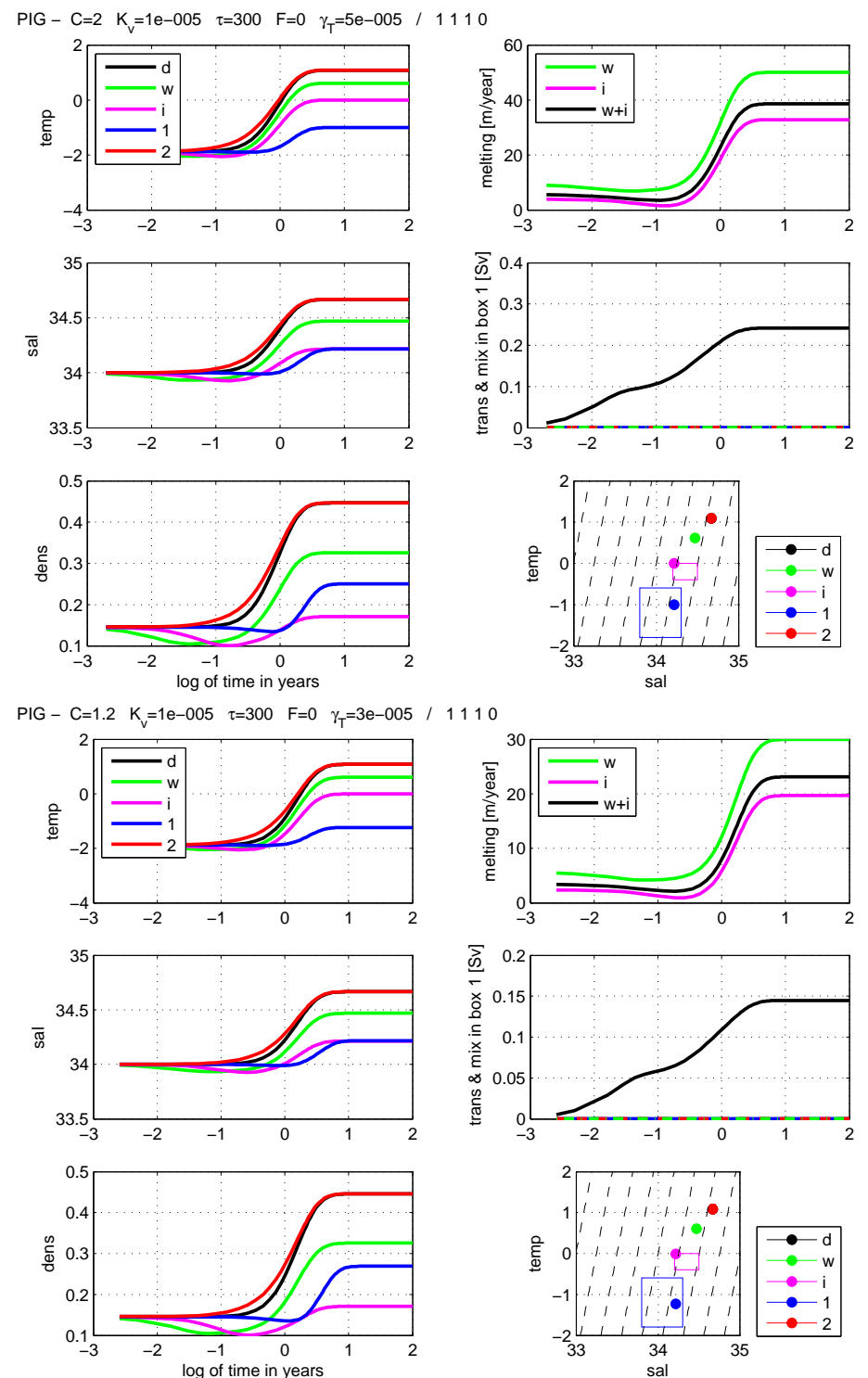

Figure 4: Two integrations for the PIG cavern with different parameters. Each experiment is displayed in 6 panels, showing the development of temperature, salinity, density (relative to a state with zero temperature and $34 \mathrm{psu})$, melting rate, overturning, and a $T / S$-diagram of the final steady state. The rectangles in this diagram indicate a rough estimate of the range of observed hydrographic parameters in the frontal boxes for the particular cavern. Note the difference in the axes scales of corresponding panels. Upper panel: $C=2$, otherwise standard parameters. Lower panel: $C=$ $1.2, \gamma_{T}=\gamma_{S} / 0.04=3 \times 10^{-5} \mathrm{~ms}^{-1}$.

box 1 (moves the blue dot in the $T / S$-diagram to to higher or lower temperatures) but has no effect on outflow box $i$. Likewise, less or more mixing (e.g. to still a moderate size of 

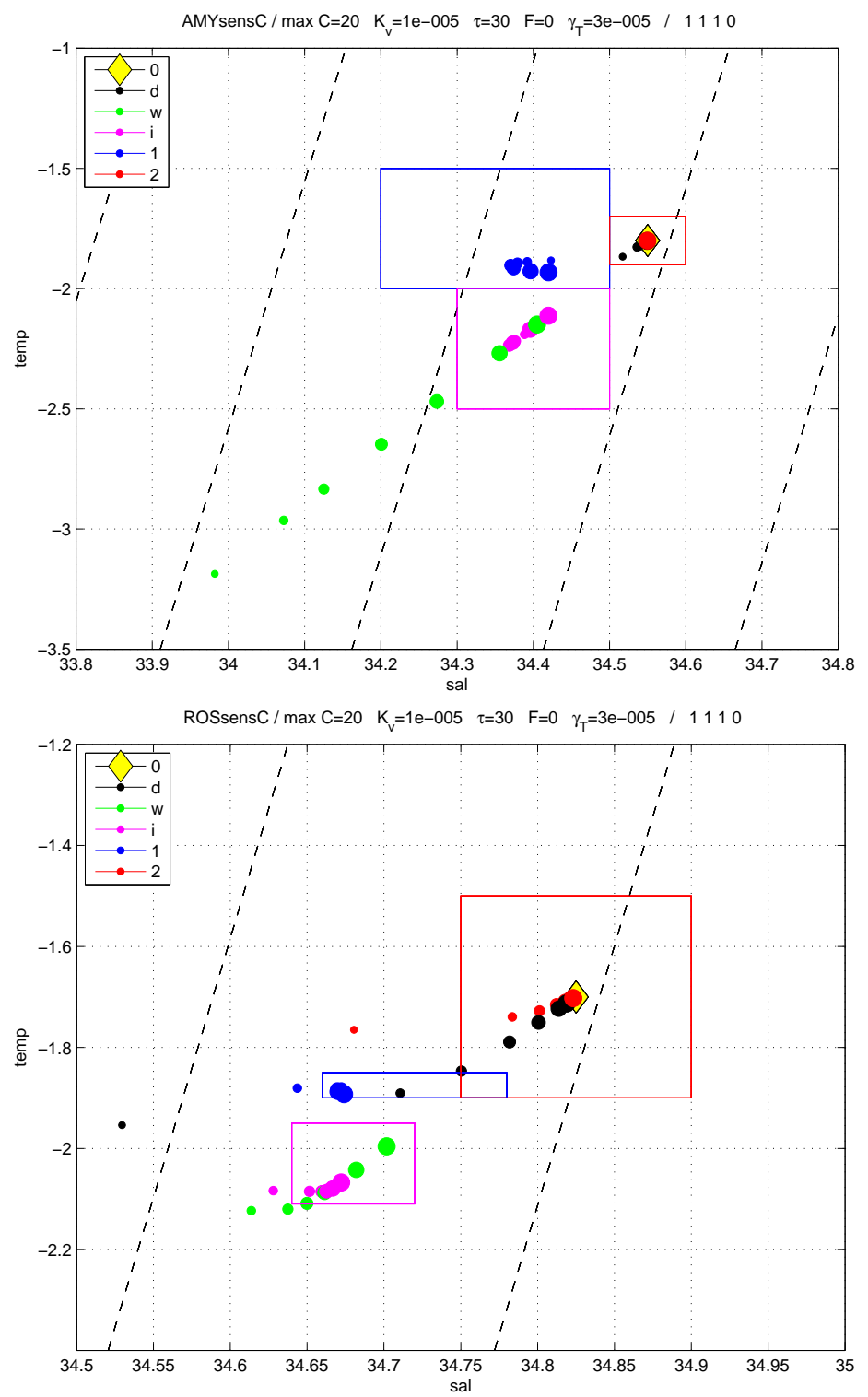

Figure 5: Sensitivity experiments for $A M Y$ and ROS, showing the TS diagrams for seven $C$ values. $C$ changed according to $[0.1,0.3,0.5,1,2,5,10] \times C_{0}$ with $C_{0}=2$. All other parameters are identical for the experiments, as given in Table 2, except for the modified turbulent exchange coefficients (see text). The inflow temperature $T_{0}$ and salinity $S_{0}$ are chosen as the middle point of the margins of $T_{2}, S_{2}$ from Table 4. The different experiments are displayed by varying dot sizes, increasing from the low $C$ value to the high one.

$K_{v}=10^{-4} \mathrm{~m}^{2} \mathrm{~s}^{-2}$ ) has very little effect, and increasing $F_{1}$ mainly influences the salinity of box 1. With the higher amount of salt in this upper front box, however, the system gets easily unstable, sometimes only for a short period until the melting in the cavern and 
advection leads to a reduction of the salinity. A major change is expected for a decreased or enhanced circulation, modeled by decreasing or increasing the value of $C$ (a smaller $C$ will make box $i$ colder and fresher, a higher $C$ warmer and saltier; see next section). As a first summary we note that the circulation should have a size with $C$ around 1 , that $F_{1}$ should indeed be small (if not zero), and that a bit of the coldness of the front surface layer should be mixed into box 1 to decrease its temperature.

In the present simulation the melting at the grounding line, $m_{w}=50.1 \mathrm{~m} / \mathrm{y}$, is likely too large but agrees with the values of the plume model by Payne et al. (2007). We felt that melt rates in excess of $30 \mathrm{~m} / \mathrm{y}$ as a net for PIG are still too large. The simulation used the standard values of $\gamma_{T}$ and $\gamma_{S}$, given in Table 2. A fine-tuning, in particular for the melting rate, can be performed by relatively moderate changes of these coupling coefficients. For $\gamma_{T}=\gamma_{S} / 0.04=3 \times 10^{-5} \mathrm{~ms}^{-1}$ we achieve $m_{m}=23.1 \mathrm{~m} / \mathrm{y}$ and $m_{i}=29.95 \mathrm{~m} / \mathrm{y}$ and still acceptable watermass characteristics for $C=1.2$ and $K_{v}, \tau, F_{1}$ as before. This experiment is displayed in the lower panels of Figure 4.

In view of the simplicity of the model we made no attempt of an objective optimization of the model parameters. In fact, they are not very robust: the change of one of them may be counteracted by a change of another one. Moreover, in most cases there is a certain range of acceptable values. Note that the consideration of the observed melt rate for the tuning, as done for PIG, will not be made for the other cavern systems. The simulated melt rates of these caverns are thus regarded as predictions from the inflow conditions.

The other caverns We applied our model to all caverns listed in Table 1 . The standard parameters from Table 2 are used, except that we take the PIG-values $\gamma_{T}=\gamma_{S} / 0.04=$ $3 \times 10^{-5} \mathrm{~ms}^{-1}$. For each cavern the specific geometry of Table 3 is implemented. The temperature $T_{0}$ and salinity $S_{0}$ of the external reservoir are chosen as the middle point of the margins of $T_{2}, S_{2}$ from Table 4 . Figure 5 exemplifies the fitting procedure (changing only $C$ ) for two caverns (AMY and ROS), and Figure 6 shows the temperatures, salinities, overturning strengths and melt rates as function of $C$ for some caverns. We assess a simulation as acceptable if the front hydrography $T_{1}, S_{1}, T_{2}, S_{2}$ and particularly $T_{i}, S_{i}$ are within their observation ranges (given in Table 4). In most cases this goal was achieved for some range of $C$ values. Similar sensitivity experiments were also made for the other basic parameters $K_{v}, \tau$, and $F_{1}$ with slight improvements of the fits but will thus not be reported here.

A few peculiarities of the individual caverns are worth mentioning. The in- and outflow values are not very accurately known for some of the caverns, making it easier to find an 'optimal' fit, but particularly for FIM and GTZ we could only find reasonable fits if the inflow temperature $T_{0}$ is changed to values well below zero. We conclude that in- and outflow characteristics have not yet been identified for these caverns. Most of the caverns show freezing in box $i$ close to the shelf ice front, at least for a slow circulation ( $\operatorname{small} C$ ) as in the case of FIM (not displayed) and ROS. Very clear freezing signals are seen for AMY, FIL and RON for all values of $C$. The melt rates cover quite a large range from well below a meter per year (FIL, FIM, RON, ROS) to the large melt rates of GTZ (not displayed) 

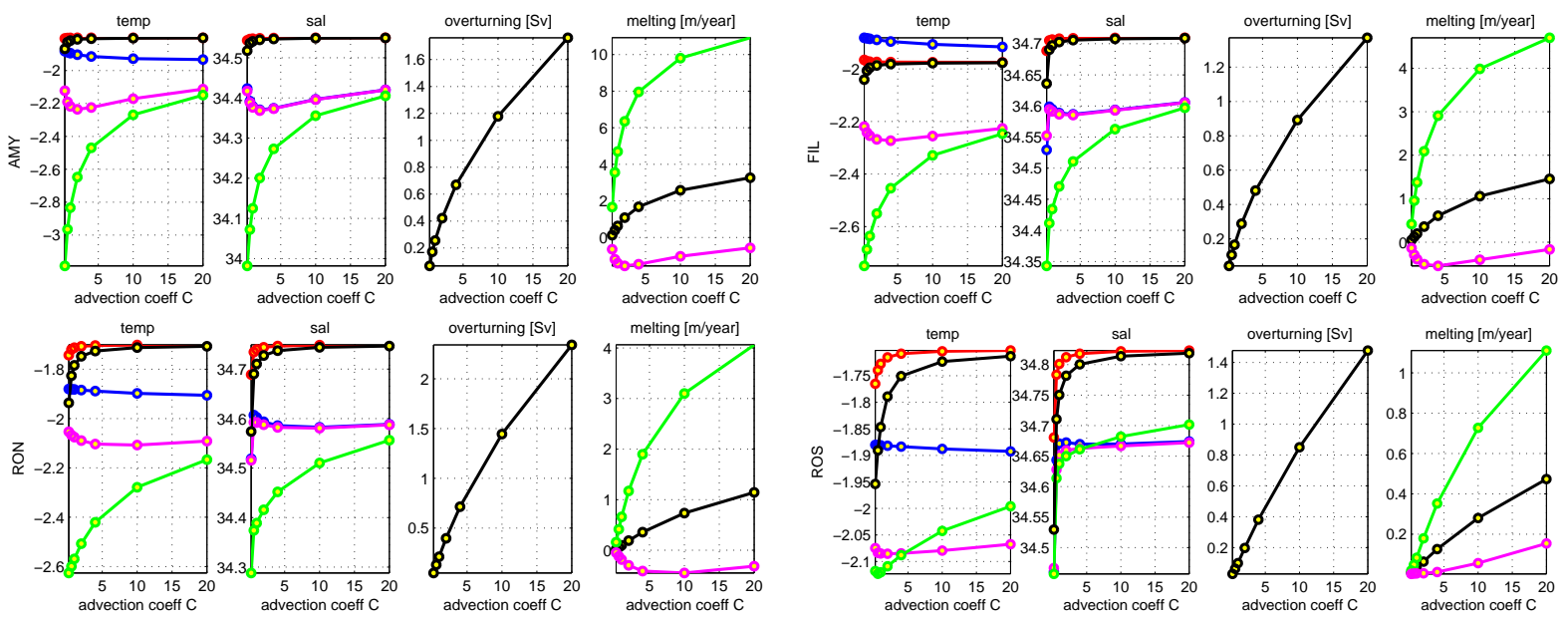

Figure 6: Sensitivity experiments for the caverns $A M Y, F I L, R O N$, and ROS. The figure shows temperature, salinity, overturning strength, and melt rate as function of $C$.

and PIG with 20 to $30 \mathrm{~m} / \mathrm{y}$. The overturning rates do not differ substantially between the seven caverns: in the cavern ensemble overturning values from $0.1 \mathrm{~Sv}$ to $1 \mathrm{~Sv}$ are found, and though in each member the overturning rate is clearly related to the respective inflow temperature, there is not such correlation obvious in the ensemble.

\section{The melting law}

The dependence of the melting rate on the inflow conditions at the front is a major concern. In particular, the variation of the grounding line melt rate $m_{w}$ with the offshore temperature $T_{0}$ of the inflowing water is of interest because $T_{0}$ is expected to increase for a warmer climate, leading to larger melting, as shown in Figure 8 below. A discussion of this issue has been given by Holland et al. (2008). For their own idealized model they find a quadratic increase of the basal melt rate as the offshore part of the ocean outside the ice shelf warms. They report as well the relationships found in previous studies, revealing (almost) linear and (almost) quadratic laws. As Holland et al. (2008) point out, 'the different modeling studies should not be intercompared'. From a limited number of experimental data an exact law cannot be detected. But even more severe are the differences of assumptions made in the models, ranging from the model codes and geometries to melting physics and warming scenarios.

Our model is designed as simple as possible. Nonlinearities arise only via advection, the quadratic equation (7) of the three-equations model, and the 'Jenkins' terms in the freezing/melting physics. In Figure 7 we show the melt rates of our numerical model for a particular geometric configuration and hydrographic conditions at the front (we take the RON configuration, see Table 3 and Table 4), resulting for a range of $T_{0}$ values. The first panel derives from the model described so far. The basal melting increases with increasing 

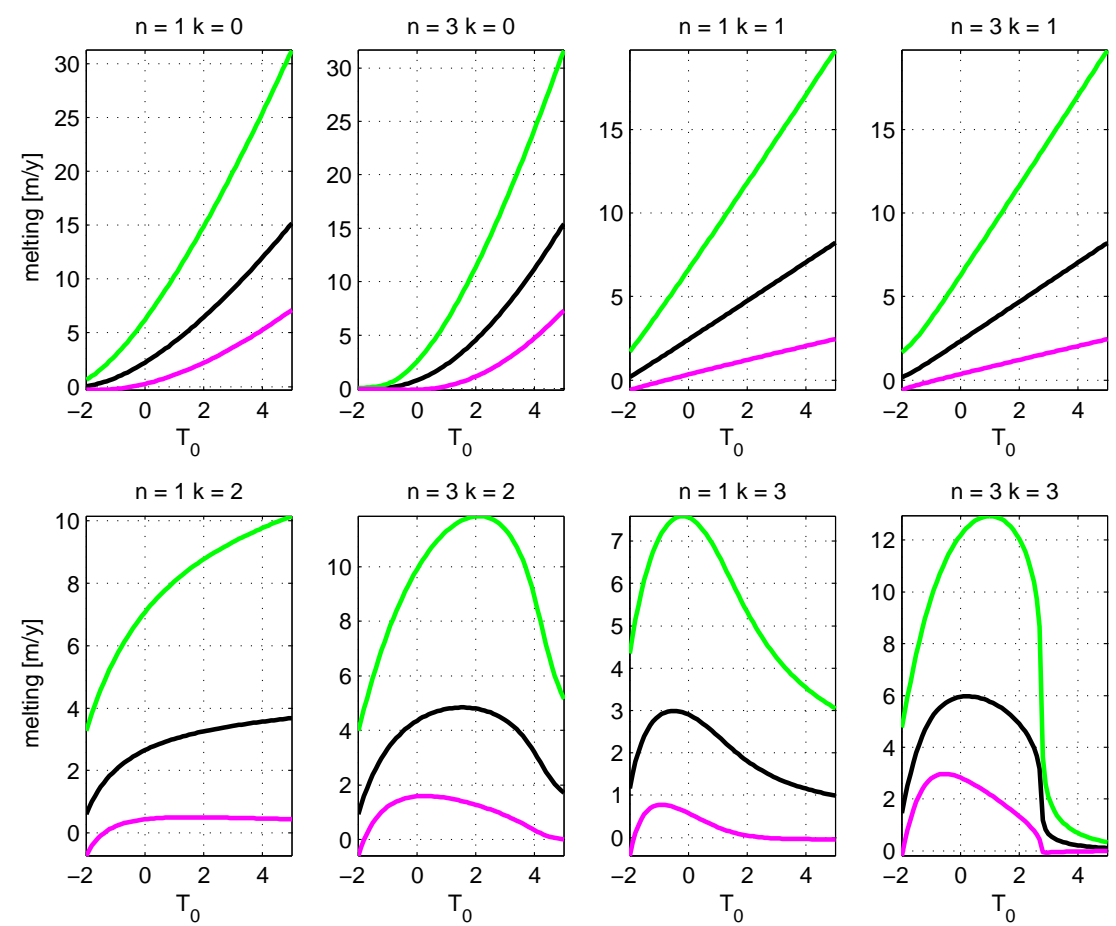

Figure 7: Melting rates obtained for various overturning relations and velocity dependencies of the turbulent exchange coefficients with power laws according to (20) and (21) In and $k$ are indicated in the title] for the RON configuration. Colors: $m_{w}$ green, $m_{i}$ magenta, $m_{m}=\left(m_{w} A_{w}+m_{i} A_{i}\right) /\left(A_{w}+A_{i}\right)$ black.

$T_{0}$, and this occurs in a nonlinear way. For the melt rates there is a shift of a power-law dependence with a higher power (likely quadratic) at small $T_{0}$ to a lower power dependence (likely linear) at higher $T_{0}$.

The other panels of Figure 7 use an extended model version with more nonlinearities implemented: at first, a power law dependence on the density difference is assumed for the strength of the overturning rate

$$
q \sim\left|\rho_{2}-\rho_{w}\right|^{n-1}\left(\rho_{2}-\rho_{w}\right)
$$

Furthermore, we consider the turbulent coefficients of the basal freezing/melting layer as dependent on the velocity with the simple relation

$$
\gamma_{T S}=\tilde{\gamma}_{T S}\left|q / q_{c}\right|^{k}
$$

with a constant $q_{c}$ and the tilded coefficients given by the values we used so far. Our standard model is thus $n=1, k=0$. The cases $n=1,3$ combined with $k=0,1,2,3$ and $q_{c}=1 \mathrm{~Sv}$ are displayed in the panels of Figure 7 . It becomes obvious that a wide range of dependencies can be generated by a suitable combination of the above parameterizations. 
In particular, there is shift of regimes between the nonlinearity of advection and the nonlinearity of the melting entrainment. Note that the velocity dependence does carry over to the temperature and salinity in the turbulent layer, but it enters the melting rate. When the melting rate gets a higher $q$-dependence than the advection (for $k>1$ ) an increase of the frontal temperatures implies a decrease of melting.

Nonlinear relations between $q$ and $\rho_{2}-\rho_{w}$ are likely if the meridional density torque in the cavern is balanced by bottom formstress rather than linear friction. An even more complicated behavior may be expected if the turbulent exchange coefficients are considered to be a function of the flow velocity, as proposed by Jenkins and Bombosch (1995) and used e.g. by Holland et al. (2008). We conclude that a unique melting law can unlikely be found from a suite of numerical models with different physics, as analyzed in the latter study. In the real world, caverns may of coarse differ with respect to appropriate parameterizations, i.e. they may work with different $n$ and $k$.

\section{Discussion and conclusions}

We have developed a simple model for a fast assessment of the strength of the overturning circulation and the melting rate in an ice shelf cavern. While the resolution with a few boxes is extremely coarse, the melting formulation (the three-equations module of Hellmer and Olbers 1989) is that used in high-resolution three-dimensional models. In the simplest version of our model the overturning strength is parameterized by a linear friction law, motivated by the balance of vorticity diffusion and the driving gradient of density between the front and the grounding line of the respective cavern. The coefficient of proportionality and a few further model parameters (describing vertical mixing in the cavern, and the turbulent exchange processes at the ice shelf base) are tuned by fitting to the hydrographic parameters (temperature and salinity in in- and outflow cores) observed at the ice shelf front. The model is able to reproduce the melting and freezing zones along the ice shelf base and predicts reasonable values of melt rates in seven caverns which were chosen as a representative ensemble of Antarctic ice shelves and glaciers.

The response to global warming, assuming that just the inflowing temperature $T_{0}$ increases, is substantial. Referring to Figure 8 , a $1^{\circ} \mathrm{C}$ warming leads to an increase of the total melting rate by a factor of about 1.6 for PIG (the factor is the minimum in the ensemble) and 13.7 for ROS (maximum in the ensemble) for the standard model version. The figure also displays the very similar result obtained with a model version in which the turbulent exchange coefficients are velocity dependent. The figure thus exemplifies the sensitivity of the model to a change of the physics. The model, of course, cannot account for a possible change in the basal area which might occur in a warmed state.

By numerical solutions of the cavern model and by analytical treatment we find a linearquadratic melting law for the dependence of the melting rate on the inflow temperature $T_{0}$ : quadratic at low $T_{0}$ and linear at large $T_{0}$. Implementing, however, further nonlinearities into the governing equations - e.g. a nonlinear relation between the overturning rate and the density gradient, or a velocity dependence of the turbulent exchange coefficients - the 


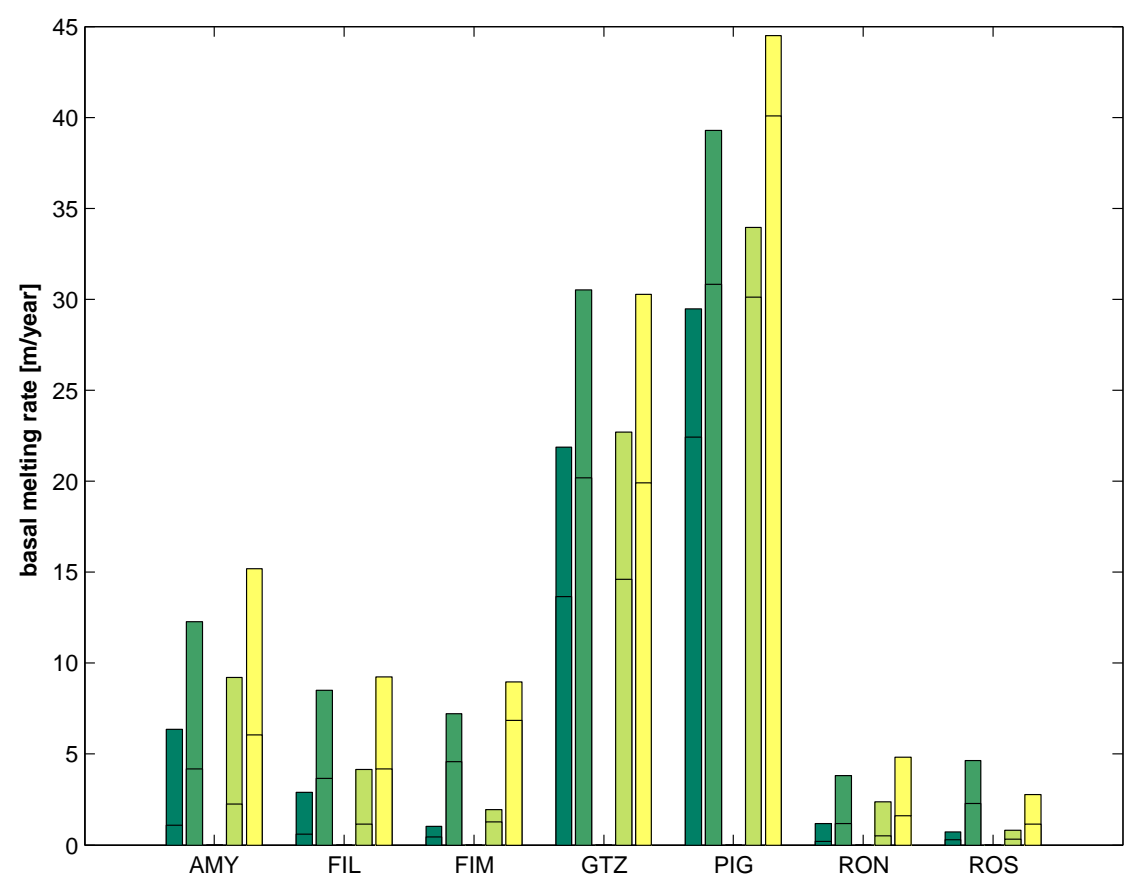

Figure 8: Basal melt rates for all caverns, evaluated for the respective 'optimal' $C$ values. For each cavern the left two columns [greenish] refer to our standard model with linear overturning rate and constant turbulent exchange coefficients (in the notation of section 4 this is $n=1, k=0$ ), the right two columns [yellowish] to a model version with extended nonlinearities $(n=1, k=1)$. For each model version the first column is for standard $T_{0}$, the second column for $T_{0}+1^{\circ} \mathrm{C}$. In each column, the lower block applies to area mean melting $m_{m}$ and the total block to melting $m_{w}$ in the grounding line box.

model yields quite different melting laws.

The model can be further simplified concerning its structure, e.g. combining box 2 with the almost passive box $d$ (see Figure 1), or extended by introducting more boxes to achieve a better resolved cavern geometry. The basic parameterization of the overturning is not easily improved if one wants to remain in the realm of box models: the coupling of the overturning strength to the density gradient is an unavoidable model crook. We intend to couple our box model to a global OGCM, implementing a few major caverns, and study the influence of the outflowing cold watermasses on the circulation, both locally around Antarctica and remotely in the AABW branches penetrating the world ocean abyss.

Acknowledgements We appreciate the very useful comments and critics of Adrian Jenkins.

\section{References}

Braun, M., A. Humbert, and A. Moll. Changes of Wilkins Ice Shelf over the past 15 years and 
inferences on its stability. The Cryosphere Discuss., 2:341-382, 2008.

De Angelis, H. and P. Skvarca. Glacier surge after ice shelf collapse. Science, 299(5612):15601562, March 2003.

Dinniman, M. S. and Klinck, J. M. and W. O. Smith. Influence of sea ice cover and icebergs on circulation and water mass formation in a numerical circulation model of the Ross Sea, Antarctica. Journal of Geophysical Research-Oceans, 112, 2007.

Grosfeld, K. and Schröder, M. and Fahrbach, E. and Gerdes, R. and A. Mackensen. How iceberg calving and grounding change the circulation and hydrography in the Filchner Ice ShelfOcean System. Journal of Geophysical Research-Oceans, 106(C5):9039-9055, 2001.

Grosfeld, K., Hellmer, H.H., Jonas, M., Sandhäger, H., Schute, M., and D.G. Vaughan. Marine ice beneath Filcher Ice Shelf: evidence from a multi-disciplinary approach. In S. S. Jacobs and R. Weiss, editors, Ocean, Ice and Atmosphere: Interactions at Antarctic Continental Margin, volume 75 of Antarctic Research Series. American Geophysical Union, 1998.

Hellmer, H. H. Impact of Antarctic ice shelf melting on sea ice and deep ocean properties. Geophys. Res. Lett., 31:L10307, doi:10.1029/2004GL19506, 2004.

Hellmer, H.H., and D. Olbers. A two-dimensional model for the thermohaline circulation under an ice shelf. Antarctic Science, 1:325-336, 1989.

Hellmer, H.H., Jacobs, S.S., and A. Jenkins. Oceanic erosion of a floating Antarctic glacier in the Amundsen Sea. In S. S. Jacobs and R. Weiss, editors, Ocean, Ice and Atmosphere: Interactions at Antarctic Continental Margin, volume 75 of Antarctic Research Series, pages 83-100. American Geophysical Union, 1998.

Holland, P. R. and Jenkins, A. and D. M. Holland. The response of ice shelf basal melting to variations in ocean temperature. Journal of Climate, 21(11):2558-2572, 2008.

IPCC, Contribution of working group I to the Fourth Assessment Report of the Intergovernmental Panel on Climate Change. Climate change 2007: The physical science basis (summary for policymakers). IPCC Secretariat, Geneva, Switzerland, 2007.

Jacobs, S., and C.F. Giulivi. Interannual ocean and sea ice variability in the Ross Sea. In S. S. Jacobs and R. Weiss, editors, Ocean, Ice and Atmosphere: Interactions at Antarctic Continental Margin, volume 75 of Antarctic Research Series. American Geophysical Union, 1998.

Jacobs, S. S., H. H. Hellmer, C. S. M. Doake, A. Jenkins, and R. M. Frolich. Melting of ice shelves and the mass balance of Antarctica. J. Glaciol., 38:375-387, 1992.

Jenkins, A., and A. Bombosch. Modeling the effect of frazil ice crystals on the dynamics and thermodynamics of ice shelf water plumes. J. Geophys. Res., 100:6067-6981, 1995.

Jenkins, A. and Hellmer, H. H. and D. M. Holland. The role of meltwater advection in the formulation of conservative boundary conditions at an ice-ocean interface. Journal of Physical Oceanography, 31(1):285-296, 2001.

Joughin, I. and L. Padman. Melting and freezing beneath Filchner-Ronne Ice Shelf, Antarctica. Geophysical Research Letters, 30(9):1477, 2003.

McPhee, M.G. Turbulent heat flux in the upper ocean under sea ice. J. Geophys. Res., 97:5365$5379,1992$.

Nicholls, K. W., Abrahamsen, E. P., Buck, J. J. H., Dodd, P. A., Goldblatt, C., Griffiths, G., Heywood, K. J., Hughes, N. E., Kaletzky, A., Lane-Serff, G. F., McPhail, S. D., Millard, N. W., Oliver, K. I. C., Perrett, J.,Price, M. R., Pudsey, C. J., Saw, K., Stansfield, K., Stott, M. J., Wadhams, P., Webb, A. T., and J. P. Wilkinson. Measurements beneath an 
Antarctic ice shelf using an autonomous underwater vehicle. Geophysical Research Letters, 33(8):L08612, 2006.

Nicholls, K. W.,Padman, L., Schröder, M., Woodgate, R. A., Jenkins, A., and S. Osterhus. Water mass modification over the continental shelf north of Ronne Ice Shelf, Antarctica. Journal of Geophysical Research-Oceans, 108(C8):3260, 2003.

Olbers, D., and Zhang, J. The global thermohaline circulation in box and spectral low-order models. Part 1: single basin models. Ocean Dynamics, 58(3-4):311-334, 2008.

Payne, A. J., Holland, P. R., Shepherd, A. P., Rutt, I. C., Jenkins, A., and I. Joughin. Numerical modeling of ocean-ice interactions under Pine Island Bay's ice shelf. Journal of Geophysical Research-Oceans, 112(C10):C10019, October 2007.

Rignot, E. Changes in West Antarctic ice stream dynamics observed with ALOS PALSAR data. Geophysical Research Letters, 35(12):L12505, 2008.

Rignot, E. and Jacobs, S. S. Rapid bottom melting widespread near Antarctic ice sheet grounding lines. Science, 296(5575):2020-2023, 2002.

Skvarca, P., Rack, W.,Rott, H., and T. I. Y. Donangelo. Climatic trend and the retreat and disintegration of ice shelves on the Antarctic Peninsula: an overview. Polar Research, 18(2):151157, 1999.

Smedsrud, L. H., Jenkins, A.,Holland, D. M., and O. A. Nost. Modeling ocean processes below Fimbulisen, Antarctica. Journal Of Geophysical Research-Oceans, 111(C1):C01007, 2006.

Stommel, H. Thermohaline convection with two stable regimes of flow. Tellus, 13:224-230, 1961.

Thoma, M., A. Jenkins, D. Holland, and S. Jacobs. Modelling Circumpolar Deep Water intrusions on the Amundsen Sea continental shelf, Antarctica. Geophys. Res. Lett., 35:L18602, doi:10.1029/2008GL034939, 2008.

Williams, M. J. M. and Grosfeld, K. and Warner, R. C. and Gerdes, R. and Determann, J. Ocean circulation and ice-ocean interaction beneath the Amery Ice Shelf, Antarctica. Journal of Geophysical Research-Oceans, 106(C10):22383-22399, 2001.

Wingham, D. J. and Shepherd, A. and Muir, A. and Marshall, G. J. Mass balance of the Antarctic ice sheet. Philosophical Transactions Of The Royal Society A-Mathematical Physical And Engineering Sciences, 364(1844):1627-1635, 2006.

Wong, A.P.S., N.L. Bindoff, and A. Forbes. Ocean-ice shelf interaction and possible bottom water formation in Prydz Bay, Antarctica. In S. S. Jacobs and R. Weiss, editors, Ocean, Ice and Atmosphere: Interactions at Antarctic Continental Margin, volume 75 of Antarctic Research Series, pages 173-187. American Geophysical Union, 1998.

Wright, D.G., Stocker, T.F., and D. Mercer. Closures used in zonally averaged ocean models. J. Phys. Oceanogr., 28:791-804, 1998.

\section{Appendix}

In section 2 we have computed the melting rate as function of the ambient cavern temperature $T_{w}$ and salinity $S_{w}$. Here we make the attempt to express it as a function of the temperature $T_{0}$ and salinity $S_{0}$ of the water flowing into the cavern at the front. For simplicity we neglect the diffusive terms in the thermohaline balances. Summing the steady state balances for the boxes $2, d$, and $w$ we arrive at 

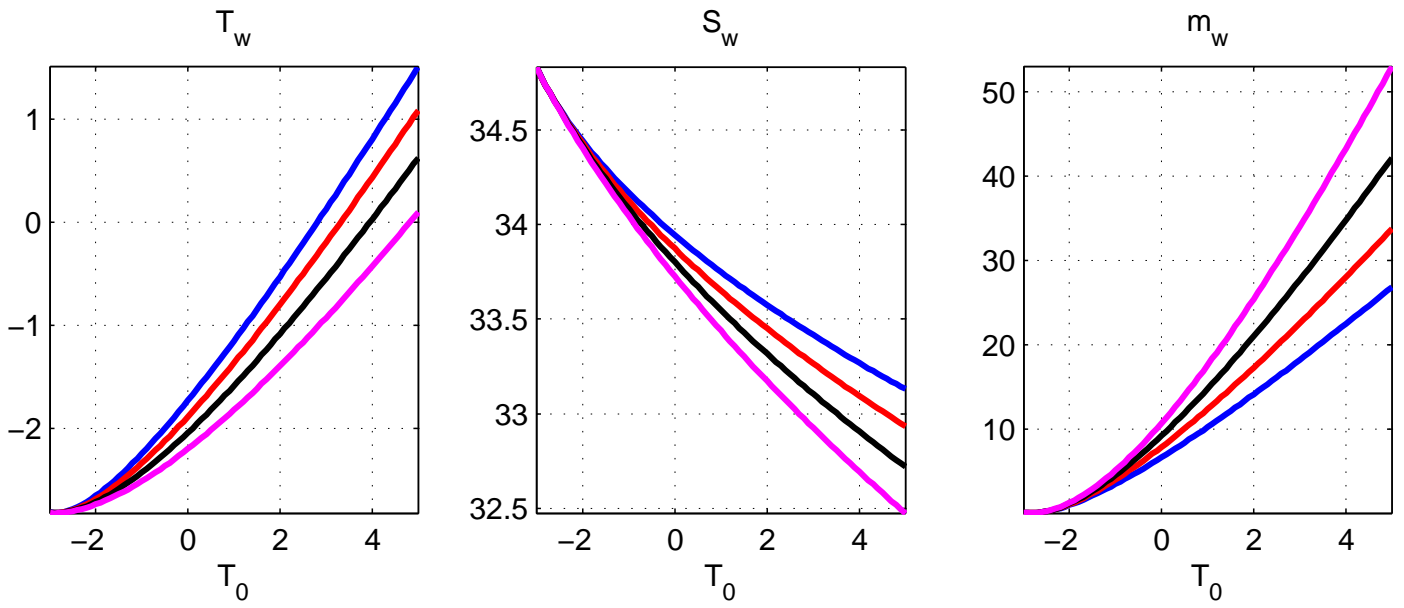

Figure 9: $\quad T_{w}, S_{w}$ and melting rate $m_{w}$ as function of $T_{0}$, calculated from (26) and the values $C=2, A_{w}=1.1 \times 10^{11} \mathrm{~m}^{2}$ (appropriate for RON). The turbulent exchange coefficient $\gamma_{T}^{\star}$ is changed according to $[0.5,0.7,1,1.5] \times 3.5 \times 10^{-5} \mathrm{~ms}^{-1}$ shown by the blue, red, black, and magenta curves, respectively.

$$
\begin{aligned}
q\left(T_{0}-T_{w}\right)+A_{w} m_{w}\left(T_{b w}-T_{w}-\nu \lambda\right) & =0 \\
q\left(S_{0}-S_{w}\right)+A_{w} m_{w}\left((1-\nu) S_{b w}-S_{w}\right) & =0
\end{aligned}
$$

where (6) has been used. Inserting now $q=C\left(\rho_{2}-\rho_{w}\right)$ with $\rho_{2}=\rho_{0}$ and the threeequations relation (7), we arrive at a set of equations for $T_{w}$ and $S_{w}$ which defies analytical treatment. Some reasonable approximations, however, lead to a manageable problem. First, $T_{b w}-T_{w} \ll \lambda$ and $(1-\nu) S_{b w} \ll S_{w}$ are valid. Secondly, the melting physics can be linearized, as proposed by McPhee (1992). The freezing law (5) is applied with the salinity $S_{b w}$ in the turbulent layer replaced by the ocean salinity $S_{w}$ outside the layer, i.e. $T_{b w}=a S_{w}+b-c p_{w}$. At the same time a slightly modified coefficient $\gamma_{T}^{\star}$ is used in the first equation of (6) to fit the nonlinear laws of the three-equations model. The melting rate becomes

$$
m_{w}=-\frac{\gamma_{T}^{\star}}{\nu \lambda}\left(a S_{w}+b-c p_{w}-T_{w}\right)
$$

With the abbreviations $x=T_{0}-T_{w}, y=S_{0}-S_{w}, g_{1}=A_{w} \gamma_{T}^{\star} / C \rho_{*}, g_{2}=g_{1} / \nu \lambda, T^{\star}=$ $a S_{0}+b-c p_{w}-T_{0}$ the equations (22) become, after the mentioned approximations,

$$
\begin{aligned}
-\alpha x^{2}+\beta y x+g_{1}\left(T^{\star}+x-a y\right) & =0 \\
\alpha x y-\beta y^{2}-g_{2}\left(S_{0}-y\right)\left(T^{\star}+x-a y\right) & =0
\end{aligned}
$$


implying $y=x S_{0} /(\nu \lambda+x) \approx x S_{0} / \nu \lambda$ because $\nu \lambda \gg x$. A quadratic problem is obtained for $x$, namely

$$
(\beta s-\alpha) x^{2}+g_{1}\left(T^{\star}+x(1-a s)\right)=0
$$

with $s=S_{0} / \nu \lambda$. Furthermore, as $\ll 1$ so that $(\beta s-\alpha) x^{2}+g_{1}\left(T^{\star}+x\right)=0$. Proper expansion reveals that $T_{w}=T-x$ is quadratic for small $T_{0}$ and linear for large $T_{0}$. The results, computing $T_{w}, S_{w}$ and $m_{w}$ from (26) as function of $T_{0}$, are displayed in Figure 9 for four values of the exchange coefficient $\gamma_{T}^{\star}$. The configuration of RON has been used and the performance can be checked by comparison with the upper left panel (green curve) of Figure 7. Obviously, a suitable choice for $\gamma_{T}^{\star}$ lies between the value of the blue and red curves.

We may proceed to solve for the $i$-box properties $T_{i}, S_{i}$ and $m_{i}$, which is even simpler because the overturning strength $q$ is known now from the $w$-box solution. In fact, an analytical solution of the complete model can thus be given, even with the diffusion terms retained but based on the above described simplified freezing law. 